\title{
Epidemiology of Needle Stick-Sharp Injuries (NSSIs) and Potential High Risk Exposures among Health Professionals in Ethiopia: Neglected Public Health Concern
}

\author{
Zelalem Desalegn", Solomon Gebreselassie, Yehenew Asemamaw \\ Department of Microbiology, Immunology and Parasitology, School of Medicine, Addis Ababa University, Addis Ababa, Ethiopia \\ E-mail address: \\ tzollove@gmail.com (Z. Desalegn), solomongst@yahoo.com(S. Gebreselassie), yeh_kiam@yahoo.com (Y. Asemamaw)
}

\section{To cite this article:}

Zelalem Desalegn, Solomon Gebreselassie, Yehenew Asemamaw. Epidemiology of Needle Stick-Sharp Injuries (NSSIs) and Potential High Risk Exposures among Health Professionals in Ethiopia: Neglected Public Health Concern. American Journal of HealthResearch. Vol. 3, No. 5, 2015, pp. 298-304.doi: 10.11648/j.ajhr.20150305.16

\begin{abstract}
Health professionals are exposed to a wide range of hazards in the workplace. Needle stick injuries have been recognized as one of the occupational hazards. Healthcare worker handling sharp devices or equipment is at risk of occupational exposure to blood borne pathogens. Despite the burden of potential exposures, in Ethiopia, there are only few researches that have been conducted; as a result there is clearly paucity of information on this regard. The aim of the research conducted was to determine the epidemiology of needle stick-sharp injuries and high risk exposures among health professionals in public hospitals, Addis Ababa, Ethiopia. Hospital based cross sectional survey conducted among health professionals at public hospitals, Addis Ababa, Ethiopia. A pretested and structured questionnaire was utilized to collect data on socio-demographic, needle stick injury and other high risk exposures. Data was analyzed using SPSS version 16.Statistical significance was declared at P-value $<=0.05$. Of the total study participants, prevalence of sustained needle stick injuries (NSIs) and sharp injury was found $155(61.2 \%)$ and $127(50 \%)$, respectively. Majority of the study subjects, which account $184(72.4 \%)$ and $153(60.2 \%)$ of them were exposed for blood while ungloved and body fluid, respectively. Consistent use of gloves was reported by $52.4 \%$ of respondents. Of the total study participants, $9(3.5 \%)$ of respondents were vaccinated against hepatitis B virus infection. The study declared that exposure for potentially infectious body fluids including blood, needle stick injuries, sharp injury and other risk factors was high. But, the study indicated only very small percentages of health professionals were partially vaccinated for HBV. Taking into account the chance of potential exposure, there is a need to focus efforts on mitigating blood borne pathogen transmission through making the work place environment safe and making use of the available vaccine by vaccinating all health care workers at the start of their career.
\end{abstract}

Keywords: Health Professionals, Universal Precaution, Needle Stick Injury, Sharp Injury, Body Fluid Exposure, Vaccination Status, Ethiopia

\section{Introduction}

Health care workers are exposed to a wide range of hazards in the workplace. Needle stick injuries (NSIs) have been recognized as one of the occupational hazards. Previous study revealed that it was the most common source of blood exposure reported (58\%), followed by non-intact-skin and mucous membrane contamination $(22.7 \%$ and $11.2 \%$ respectively) and cuts ( $8 \%$ ) [1].Needle stick injuries are a big problem. In the United States, CDC estimates indicate that 600,000 to 1 million such injuries occur annually. About half of these injuries go unreported [2,3].
Needle stick injuries (NSIs) are considered as common occupational hazards for Health Care Workers (HCWs). However, available statistics underestimate the severity of the problem because many HCWs do not report their injuries. Therefore, it is not known how serious the problem is or how well prevention programme will work. Universal precautions and care to avoid injuries with sharps and instruments have been implemented to decrease the risk and prevent infection. In addition, immunization with the HBV vaccine is recommended as an important adjunct to universal precautions [4].

With regard to prevention, when exposures occur, the risk of infection can be significantly reduced by following 
protocols for PEP. Guidelines have been issued for the management of HCWs who have had occupational exposure to blood borne pathogens. This includes urgent evaluation of the source and exposed person's status along with the timely administration of hepatitis B immune globulin (HBIG), hepatitis B vaccine and/or HIV PEP where applicable. For $\mathrm{HCV}$, testing should be performed to determine if infection develops [5-9].

Any healthcare worker handling sharp devices or equipment such as scalpels, sutures, hypodermic needles, blood collection devices, or phlebotomy devices is at risk of occupational exposure to blood borne pathogens. Certain groups of individuals are at greater risk than others because of the nature of their work. Such is the case with doctors, nurses and medical students where their responsibilities necessarily involve the risk of exposure to patient's blood.

In Ethiopia very limited data is available on needle stick sharp injuries exposure status among health professionals. This current study was conducted among health professionals to determine the epidemiology of needle stick -sharp injuries and other high risk exposures at two hospitals in Addis Ababa, Ethiopia. As the incidence of blood borne pathogen continues to rise, increasing attention to the risks of needle stick injury to health care workers would seem appropriate. Since this paper is the first in its kind in the mentioned research area, this will give baseline information about the issue and will give future insights for prevention and intervention measures. In addition, high risk groups like HCWs would understand the extent of the burden and give due emphasis to adhere with personal protective equipment (PPE).

\section{Methods}

\subsection{Study Design, Area and Period}

A cross-sectional study was carried to determine the prevalence of needle stick-Sharp injuries (NSSIs) and other high risk exposures among health care workers at Addis Ababa, Ethiopia during November 2011 to January 2012.

\subsection{Sample Size Calculation and Sampling Technique}

A single population proportion formula was employed and accordingly 254 study participants were involved. To get the calculated sample size participants were approached conveniently during the study period.

\subsection{Data Collection Procedure and Instrument}

The survey research employed pretested and structured questionnaire. The questionnaire used was constructed based on questionnaire used in a previous study and additional relevant variables missed in literatures were considered. Ambiguous questions were revised; sequence of questions and the flow was improved. The questionnaires were divided into different parts. The first part consisted of questions on their socio-demographic characteristics. The other parts were on the prevalence study of needle stick injuries and other high risk exposures where the respondents were asked about their experience in handling needles and the prevalence of needle stick injuries. The questionnaires were administered by using face-to-face interviews to ensure a good response rate and to ensure all questions were answered.

\subsection{Data Management and Statistical Analysis}

The collected data was checked for completeness and missed value. For each questionnaire code was given to maintain confidentiality and data entry and analysis was made using SPSS soft ware. Frequency and percentage was done and the statistical analysis was performed using logistic regression. Data was analyzed and presented by tables. A P value $<=0.05$ was considered to be statistically significant.

Ethical clearance: The study protocol was reviewed and approved by the Institutional Review Board (IRB) of Addis Ababa University, Addis Ababa Ethiopia. Permission letter was obtained from the respective hospitals to conduct the research. Prior to data collection the participants were informed about the whole aim of the study and participation in study was in voluntary base. After they are informed about the objective of the research consent was obtained from the participants. The confidentiality of respondents was ensured through keeping data files and completed questionnaire under lock.

\section{Results}

\subsection{General Characteristics}

The study participants were health professionals who have been working in the respective hospitals. A total of 254 eligible health professionals were approached in the study. According to the findings, 98(38.6\%) of the participants were in the age group 20-29 years. With regard to gender distribution, 1:1 ratio was recorded. Amongst health professionals, greater than $50 \%$ of them were nurses. The Socio-demographic characteristics are shown in Table 1.

Table 1. General characteristics of health professionals at Public hospitals, Addis Ababa, Ethiopia.

\begin{tabular}{lll}
\hline Variables & Frequency & Percent (\%) \\
\hline Age group & & \\
$20-29$ & 98 & 38.6 \\
$30-39$ & 83 & 32.7 \\
$40-49$ & 43 & 16.9 \\
$50-59$ & 30 & 11.8 \\
Sex Male & 127 & 50 \\
Female & 127 & 50 \\
Marital Status & & \\
Single & 149 & 58.6 \\
Married & 100 & 39.4 \\
Divorced & 5 & 2 \\
Religion & & \\
Orthodox & 161 & 63.4 \\
Muslim & 66 & 25.9 \\
Protestant & 27 & 10.6 \\
Job Category & & \\
Physician & 65 & 25.6 \\
Nurses & 128 & 50.4 \\
\hline
\end{tabular}




\begin{tabular}{lll}
\hline Variables & Frequency & Percent (\%) \\
\hline Midwifery's & 26 & 10.2 \\
Laboratory Technician & 35 & 13.8 \\
Duration of Service (in years) & \\
$<=10$ & 165 & 65 \\
$11-20$ & 43 & 16.9 \\
$>20$ & 46 & 18.1 .9 \\
\hline
\end{tabular}

\subsection{Prevalence of Needle Sticks Injury and other Potentially High Risk Exposure}

The study finding revealed that 205(80.7\%) and $108(42.5 \%)$ of HCWS had knowledge on universal precaution guideline and trained on infection prevention, respectively.

The study tried to assess the attitude of health professional towards needle re-capping and also measures the occurrence of needle stick injury (NSI) and sharp injury. Out of the total, more than half, which is $155(61.02 \%)$ and half of $127(50 \%)$ them had experience needle stick injury and sharp injury, respectively.

Concerning the attitude toward needle recapping, $145(57.1 \%)$ of them did not have positive attitude for needle recapping. Among those who did not agree of needle recapping, only 66(45.5\%) of them were exposed for needle injection and among those who had positive attitude $89(81.6 \%)$ of health professionals were encountered needle injection. Health professionals who had positive attitude in employing needle recapping have been exposed for needle injection 5 times more likely than their counterparts $(\mathrm{AOR}=5$; 95\%CI 2.968-9.560) and also the difference was statistically significant with P-value $=0.0001$.

The study also examined the trend of post exposure reporting habit of health professionals to the responsible department or immediate staff. Of the total 155 needle injected participants, $80(54.05 \%)$ of them failed to report the case to the concerned body. 43(54.4) of the study participants indicated that negligence was the primary reason for not reporting needle stick injury. Across the groups, the largest numbers of injured participants were in the 20-29-year-old age group.

Coming to sex distribution of encountered NSI, out of the total NSI, risk of exposure for needle injection was slightly higher in males $81(52.3 \%)$ than females $74(47.7 \%)$ but statistical difference not noted.

Out of the considered health professionals, $80(51.6 \%)$ of NSIs and $72(57.02 \%)$ of sharp injuries were reported by nurse professionals followed by Physician which was $41(26.4 \%)$ and $32(25.2 \%)$, respectively. The percentage distribution of NSIs across the study participants are depicted in the figure 1.

The research explored experience of health professionals in applying gloves has been assessed. Of the 254 health professionals, $152(59.8 \%)$ of the participants were using gloves consistently whereas $102(40.2 \%)$ used nonconsistently. Of the total participants, 135 (53.2\%), $184(72.4 \%), 153(60.3 \%), 162(63.8 \%)$ of them had history contact with liver disease patients, cutaneous exposure to blood and to any of the body fluids, and had splashed blood or body fluids in their faces, respectively. Frequency of occupational and non -occupational related exposure to risk factors among health professionals is shown in Table 2. In all risk factors displayed in table 2 , nurse professionals took the uppermost risk of exposure.

Table 2. Level of exposure to Needle stick injury and other non-needle stick exposure among health professionals in Public hospitals, Addis Ababa, Ethiopia.

\begin{tabular}{llllll}
\hline Professional category & & & & & \\
\hline Type of exposure & Number of Exposures & Physn=65 & Nursen=128 & Lab. Tecn=35 & MWn=26 \\
\hline Needle stick injury & 155 & 41 & 80 & 18 & 16 \\
Sharp injury & 127 & 32 & 72 & 11 & 12 \\
Blood exposure & 184 & 45 & 98 & 22 & 19 \\
Body fluid exposure & 153 & 40 & 82 & 19 & 12 \\
\hline
\end{tabular}

The study revealed that majority of health professionals were exposed for needle stick injury and this has been shown in table 2.

\subsection{HBV Vaccination History}

This study tried to note the vaccination status of the study participants. When vaccination status of HCWs were observed, of 254 study participants, only 9(3.5\%) of them had history of vaccination against HBV. Of the vaccinated health professionals, none of them were fully vaccinated. Occupation specific vaccination status was higher among Physician 6(67\%) followed by nurse 3(33\%).

\section{Discussion}

The present study described the prevalence of needle stick injuries and other high risk exposures among Health professionals in Ethiopia public hospitals, Addis Ababa, Ethiopia.

According to different researches conducted globally, greater than 35 million $\mathrm{HCWs}$ encounter the risk of sustaining a percutaneous injury with a contaminated sharp object every year. These exposures can lead to infections with $\mathrm{HBV}, \mathrm{HCV}$, and HIV. The risk of transmission of HBV infection by NSI is between 6 and $30 \%$ for susceptible HCWs without post-exposition prophylaxis or sufficient HBV vaccination $[10,11]$.

Our study recognized approximately above half (50\%) of the study subjects were encountered NSI and sharp injury. The present study indicated that exposure for needle stick injury and sharp injury was high. The overall prevalence of NSI and sharp injury were $155(61.02 \%)$ and $127(50 \%)$ respectively. A previous research report from Addis Ababa, Ethiopia showed that 59\% and $31.1 \%$ NSI and sharp injury were recorded respectively [12] which were in agreement 
with our study finding.

Different research reports noticed that there is blood borne pathogen positivity difference when needle stick injured compared with their counterparts. This was shown by research finding from India which reported a significantly higher incidence of HBsAg occurred among those with history of frequent and occasional needle pricks as compared to those with rare or no needle pricks [13].Also the study finding was comparable with a study conducted in Pakistan Chittagong Medical College Hospital (CMCH), Chittagong, their $66 \%$ respondent were victims and all needle stick and sharp injured were positive for $\mathrm{HBsAg}[14$ ]and also in agreement with other report, which was done previously from different regions including Sub-Saharan African countries [15].

Our finding was somewhat higher than a study from Sudan which has reported $38.4 \%$ of HCWs were exposed for NSI and most of the blood borne positive cases examined among needle pricked study participants. This difference might be due to sample size and implementation of universal precaution [16].Our result was higher than a study conducted in Egypt their $35.6 \%$ of them were exposed to at least 1 needle stick injury during the past 3 months [17].

Study from Awassa, Ethiopia showed that accidental needle stick injuries sustained by health-care workers are a common occupational hazard and a public health issue in health-care settings and $30.9 \%$ of health-care workers had experienced at least one needle stick injury in the previous year [18].

Of all professional groups, nurses had the highest risk to experience NSI, sharp injury. And other high risk exposure. This might be explained by the fact that nurses are responsible for most of blood sampling and other I.V. access procedure in the hospital; in addition to that, they are more diligent in their reporting behaviors. This finding shows needle stick and sharp injuries were quite common work place accidents and indicated that exposure to blood, body fluid, NSI and sharp injury found to be a considerable burden for health care workers and indicate the common mistakes of negligence of common procedure in a hygienic way.

In addition, lack of awareness of infection control practices, lack of resources for sterilization and the purchase of new disposable equipment might be the possible explanation for high occupation related exposure. Study from Ethiopia revealed that availability of PEP had positive association with infection prevention (AOR $=6.79 ; 95 \%$ CI:2.83-17.27)[19]. Clearly, not every NSSI is preventable, but research has shown that $74 \%$ of injuries from needle stick can be prevented [20].

Several suggestions have been made for preventing and limiting sharp injuries among HCWs. These include health education, behavior change (e.g. not recapping needle, disposal related issued), introduction of barriers to protect the caregivers, safer techniques, safer devices (e.g. needleless and self-sheathing equipment), and organization factors such as improved staffing levels and implementing teaching program to educate these downstream staff and safe handling of disposal devices. Accurate post-exposure prophylaxis and follow-up should be encouraged to eliminate or minimize the risk of transmission. Active surveillance and periodic review of interventions are important aspects to reduce NSSIs in targeted high-risk occupational groups.

HCWs were potentially exposed to blood and body fluids containing transmissible diseases and are at increased risk to acquire these pathogens [21]. Hepatitis B and C are blood borne pathogens, which might be acquired occupationally. At most institutions no attempts are made to minimize direct ungloved contact with blood of HBsAg positive patients. Moreover, most HBsAg positive materials handled are not labeled as such. This study tried to measure level of exposure for blood and other body fluids. Exposure to blood and body fluid in this group of health care personnel was quite high reaching $72.8 \%$ and $60.2 \%$, respectively.

Our finding is slightly lower than previously conducted research in Ethiopia $82.3 \%$ and $60.6 \%$ for blood and other body fluids [12].This might be as a result of HCWs updated knowledge on the mode transmission of the virus or forgetting their exposure which result in underestimation. Under reporting by our HCWs could be the reason as they did not register the episodes not considering them important enough, again pointing towards their attitude and perception. The result is higher than a $64 \%$ report from Turkey [22] where awareness on universal precaution and implementation is expected to be high.

In addition, this study investigated the exposure of health professionals for body fluids (amniotic fluid, CSF, Pleural fluid and the like) other than blood which accounts $60.2 \%$.Detailed analysis on possible risk factor for blood and body fluid exposure is not done here.

Previous reports from other countries has put several possible reasons for the wide spread presence of exposure to blood and body fluid; lack of training, long working hours, working habits, and experience are some of the reasons mentioned [23].Moreover, unprotected handling of the biological specimens and different procedures in healthcare facilities are added risk factors. Some of these factors might also operate here but other study to search out the possible risk factors operating in Ethiopian health care set up is required.

Compliance to universal precaution was assessed by consistent use of glove and $152(59.8 \%)$ consistently use glove. This indicates yet there is a problem in compliance to universal precaution. Our result finding is higher than the previous study conducted in Ethiopia [12] and reports from Turkey, [24] and India (15). This might be due to change in attitude, adherence and practice of HCWs in applying standard precaution. HCWs would have been over estimate their knowledge and practices on infection prevention and compliance to standard precautions (SPs).Our current research did not incorporate reasons for not wearing personal protective equipment (PPEs). Previous study reported that HCWs were agreed on encountering shortage and ill fitted size personal protective devices and also indicated there are' no clear rules and regulation' which govern the staff, patients 
and environment.

Training on infection prevention is very important to prevent $\mathrm{HCW}$ s from being infected by blood borne disease causing organisms like HIV, HBV, HCV and the so on. Our study noticed that less than half of $(42.7 \%)$ the study subjects had training on infection prevention. The current figure is comparable with previous reports from Ethiopia, which showed that only $45.8 \%$ of the respondents said that they ever had participated in any training dedicated to infection prevention after their respective pre-service courses [25] .Also study from Awassa reported 41.8\% of HCWS were trained on infection prevention [18].Previous report from Uganda indicated that $\mathrm{HCW}$ with no raining on infection prevention were more likely to have been exposed to hepatitis B virus infection [26].

Our study indicated there is a need to continuously support, train and supervise HCWs to improve the universal precaution. This underlines the importance of the need to provide training in preventive measures for workers in healthcare facilities.

The discovery of HBV vaccines and the results obtained from their introduction constitute a land mark of great importance for medical practice. Mass immunization lowers transmission rates and hence, pushes back the average age of infection. The world's first universal vaccination program for HBV infection was launched in 1984 in Taiwan. This program reduced the overall HBsAg prevalence rate from $9.8 \%$ in 1984 to $1.3 \%$ in 1994 among children< 15 years of age [27]. Reports of previous studies support that hepatitis B immunization decreases the incidence of HBV infection [28].In May 1992, the World Health Assembly, the governing body of the World Health Organization, endorsed recommendations that all countries should have HBV vaccine integrated into their national immunization programmes[29].

Regarding vaccination, our study showed the HBV vaccination coverage in this group of HCW's was $3.5 \%$.In previous study from Ethiopia, the hepatitis $\mathrm{B}$ vaccination rate was found to be $13 \%$ and absence of vaccination was the only risk factor associated with hepatitis B infection $(p=0.001)$ [12].

The current study revealed vaccination status was poor when compared with study from Saudi Arabia their $84 \%$ of HCWs were vaccinated [30].In early Study from Egypt, about $38 \%$ of professional staffs were vaccinated [17]. A study from Low endemic countries like Iran reported about $86.4 \%$ of $\mathrm{HCWs}$ were vaccinated and the positive rate of HBsAg among vaccinated and unvaccinated HCWs was $2 \%(6 / 299)$ and $6.4 \%(3 / 47)$,respectively[31].

Study from India revealed about $42 \%$ of the HCWs had received partial or full course of vaccination against HBV and the difference in positivity among vaccinated and unvaccinated groups was highly significant $(\mathrm{P}<0.001)[13]$. Study from Sudan revealed that all the positive cases were detected among unvaccinated HCWs but there was no significant difference in HBsAg positivity [16].

The lower vaccination status is not surprising as there is no well-designed policy by Ministry of Health to vaccinate HCW's. Moreover, cost (unavailability of vaccine), knowledge, negligence and fear of side effects might be the most important barriers to vaccination or completing vaccination. Moreover, Awareness and attitude problem might be the factor responsible for lack of vaccination. While by providing necessary facilities and requiring vaccination for hospital personnel these factors could be eliminated to achieve $100 \%$ vaccination coverage. Effective prevention of $\mathrm{HBV}$ infection is mainly by vaccination to unexposed $\mathrm{HCW}$; however, acceptance of vaccine should be promoted for such high risk categories. If possible, all the HCWs should be given vaccination for HBV preferably at the start of their career.

Majority of the study participants knew about universal precautions and this might be due overestimated report from HCWS. In one study from Saudi Arabia, 43/70(61\%) of HCWS were aware of universal precaution guidelines [29]. Previously conducted research found that sharp injury was associated with universal precaution and infection prevention training [32].

Considering the high prevalence of exposures for needle stick injuries, sharp injuries and potential exposures for blood and body fluids, the future intensive training which focuses on universal precaution and infection prevention should be in place to minimize NSIs and other high risk exposures among HCWs. To reduce the occupational exposure for blood borne infection, universal precautions must be rigorously adhered to when they carry out procedures on their patients. In addition, either pre-exposure or post exposure immunoprophylaxis and vaccine should be introduced if there is for preventing and controlling terrible disease.

\section{Conclusion}

The currently carried out study showed that health professionals were highly exposed for blood, other body fluids, NSI, sharp injury and other risk factors. Despite their exposure, the research revealed that vaccination status of the study subjects was very poor when compared with other countries. Very alarmingly, only $9(3.5 \%)$ of the study participants were partially vaccinated. Regarding training on infection prevention, majority of the study participants, which accounts $57.2 \%$ of the study participants had never been attend training.

As a recommendation all the health care workers should be trained and educated regarding universal precautions i.e. blood, body fluids and tissue of all the patients should be considered potentially infectious and appropriate barrier precautions should be taken while handling these. Use of certain safety devices e.g. blunt suture needles and needle sheathing devices that eliminate recapping are recently being introduced to reduce exposure. Modification of work practices like avoiding holding tissues with bare hands during surgery and improving coordination between members of the surgical team can also help to reduce the chances of exposure. Protective equipment like gloves, 
masks, face shields and goggles can prevent exposure in situations where splashes of blood or body fluids are likely to occur. Furthermore, yearly screening of workers should be done in order to detect infection early and take preventive and therapeutic measures well in time. HCWs should take all the preventive measure to save them. But exposure prevention is still very important because of a risk of other blood borne pathogens.

\section{Authors' contributions}

ZD took leading role in coordination, study conception, design, analysis, interpretation, manuscript write up and critical review. SG and YA assisted in manuscript draft preparation, data analysis and interpretation, drafted the manuscript and critically reviewed the manuscript. All the authors' read and approved the manuscript.

\section{Acknowledgments}

The authors would like to acknowledge Addis Ababa University for funding the research. Also we are grateful for the study participants who were willing to be engaged in the study and staff of respective hospitals for providing their enthusiastic approach and help during the entire research work.

\section{References}

[1] Ippolito G, Puro V, De Carli G. The risk of job occupational human immunodefiency virus infection in health care workers.Italian Multicenter Study.The Italian Study Group on Job occupational Risk of HIV infection. Arch Intern Med. 1993 153: $1451-1458$.

[2] International Health Care Worker Safety Center. Exposure Prevention Information Network (EPINet) Data Reports. Charlottesville: University of Virginia. (online), 1998. http:// www.med.virginia.edu/medentr/centers/epinet/estimates.html.

[3] Perry J. CDC Releases National Needlestick Estimates. Advances in Exposure Prevention 2000; 5: 19. (online) http:// www.med.virginia.edu/medentr/centers/epinet/cdcestim.html

[4] Perspectives in Disease Prevention and Health Promotion Update.Universal Precautions for Prevention of Transmission of Human Immunodeficiency Virus, Hepatitis B and other Blood-borne Pathogens in Healthcare settings.MMWR 1988; 37: $377-88$.

[5] Standard supplies list. Universal Precautions, including Injection Safety: World Health Organization (WHO). 2005. www.WHO.INT/ HIV/topics/precautions.

[6] Updated US Public Health Service Guidelines for the Management of Occupational Exposure to HBV, HCV and HIV and Recommendations for Post Exposure Prophylaxis. MMWR 2001 Jun; 50 (RR11): 1-42.

[7] United Kingdom Health Departments. Guidelines on postexposure prophylaxis for healthcare workers occupationally exposed to HIV. Department of Health, London, 1997.
[8] Department of Health. HIV post-exposure prophylaxis: Guidance from the UK Chief Medical Officers Expert Advisory Group on AIDS. 2nd ed. 2004.www.advisorybodies.doh.gov.uk/eaga/publications.htm.

[9] Cullen BL, Genasi F, Symington I, Bagg J, McCreaddie M, Taylor A et al. Potential for reported needlestick injury prevention among healthcare workers through safety device usage and improvement of guideline adherence: expert panel assessment. J Hosp Infect 2006; 63: 445-51.

[10] Deisenhammer S, Radon K, Nowak D, Reichert J. Needle stick injuries during medical training. J Hosp Infect.2006;63:263-267.

[11] Rogers B, Goodno .Evaluation of interventions to prevent needle stick injuries in health care occupations. Am J Prev Med 2000; 18:90-98 .

[12] Yimer K. Serological and molecular characterization of Hepatitis B, C andD Viruses Infections among Health Professionals in Public Hospitals, Addis Ababa, Ethiopia,2003. A thesis submitted to Addis Ababa University. http://hdl.handle.net/123456789/551

[13] Asok K.K, Baghel.P.K, Shukla.C.B, Jain.M.K.Prevalence of HBsAg among HCWs. Indian Journal of Community Medicine 2000; 25(2)

[14] Morshad MG and Howlader MAR .Incidence of hepatitis B among healthcare professionals due to medical waste handling.J Dhaka Med Coll 2009;18(2) : 140-143

[15] Kermode M, Jolley D, Langkham B .Compliance with Universal/Standard Precautions among HCWs in rural north India. Am J Infect Control 2005;33:27-33.

[16] Nail A, EltiganniS , Imam A. Seroprevalence of Hepatitis Band $\mathrm{C}$ among health care workers in Omdurman, Sudan. Sudan Journal of Medical Sciences2008; 3(3):201-206

[17] Talaat M, Walid El-Shoubary W etal.Occupational exposure to needlestick injuries and hepatitis $\mathrm{B}$ vaccination coverage among health care workers in Egypt. American Journal of Infection Control(AJIC).2003; 3(8): 469-474.

[18] Tadesse M, Tadesse T. Epidemiology of needlestick injuries among health-care workers in Awassa City, Southern Ethiopia. Trop Doct2010;40(2):111-3.

[19] Kelemua Gulilat, Gebeyaw Tiruneh. Assessment of Knowledge, Attitude And Practice of Health Care Workerson Infection Prevention in Health Institution Bahir Dar City Administration. Science Journal of Public Health. Vol. 2, No. 5, 2014, pp. 384-393

[20] Castello A, Vallino A, Argentero P, Zotti C. Prevent-ability of percutaneous injuries in HCWS: ayear-long survey in Italy. J Hosp Infect .2003;55: 290-294.

[21] CatalaniC, Biggeri A, Gottard A et al . Prevalence ofHCV infection among health care workers in a hospital in central Italy. Eur J Epidemiol 2004;19: 73-7.

[22] Azap A, Ergonul O, MemikogluKe t al.Occupational exposure to blood and body fluids among health care workers inAnkara, Turkey. Am J Infect Control .2005;33: 48-52.

[23] NsubugaFredrich M, JaakkolaMaritta S. Needlestick injuries among nurses in subSaharan Africa. Tropical Medicine and International Health 2005;10:773-81. 
[24] Kosgeroglu N and Ayranci U. Needle stick and sharps injuries among nurses in the healthcare sector in a city of western Turkey. J Hosp Infect 2004; 58: 216-23.

[25] DamteM .Assessement of KAP of HCWS on Universal Precaution in North Wollo Zone, Amhara Region, North Eastern Ethiopia. A forth MPH Extract published by EPHA 2008 ;Extract No (4): 20-26

[26] Ziraba A, Bwogi J, Namale A et al. Sero-prevalence and risk factors for HBV infection among HCWs in a tertiary hospital in Uganda. BMC Infectious Diseases 2010;.10:191.

[27] Ni YH, Chang MH,Hang LM.HBVinfection in children and adolescents in a hyper endemic area: 15 years after mass hepatitis B vaccination. Ann intern Med 2001; 135:796-800.

[28] Jefferson T, Demicheli V, DeeksJ.Vaccines for preventing hepatitis B in health-care workers. Cochrane Database Syst 2000;Rev 2: CD00010
[29] KaneM. Global program for control of hepatitis B infection. Vaccine 1995; 13 ( Suppl.1 ): 4 -9

[30] MaqbulAlam(2002). A study conducted on knowledge, attitude and practices among health care workers on NSIs, Saudi Arabia. Annals of Saudi Medicine 2002; Vol 22, Nos 5-6

[31] Mirza Khalil .B, Ayyoob .K, Adel M, Eslam G. Seroprevalence of HBV infection and vaccination compliance among HCWS in Fars Province, Iran. Iranian Journal of Clinical InfectiousDiseases.2010;5(1):45-50

[32] Berhanu Elfu Feleke. Prevalence and Determinant Factors for Sharp Injuries among Addis Ababa HospitalsHealth Professionals. Science Journal of Public Health. Vol. 1(5) 2013;189-193. doi: 10.11648/j.sjph.20130105.11 